\title{
Enhancement of thermal transport in the degenerate periodic Anderson model
}

\author{
V. Zlatici ${ }^{1,2}$, R. Monnier ${ }^{3}$, J. K. Freericks ${ }^{4}$ \\ ${ }^{1}$ Institute of Physics, Bijenička c. 46, 10001 Zagreb, Croatia (permanent address), and \\ ${ }^{2}$ International School for Advanced Studies (SISSA), Via Beirut 2-4, I-34014 Trieste, Italy \\ ${ }^{3}$ ETH Hönggerberg, Laboratorium für Festkörperphysik, 8093 Zürich, Switzerland and \\ ${ }^{4}$ Georgetown University, Washington D.C., USA
}

(Dated: August 8, 2018)

\begin{abstract}
The low-temperature transport coefficients of the degenerate periodic $S U(N)$ Anderson model are calculated in the limit of infinite correlation between $f$ electrons, within the framework of dynamical mean-field theory. We establish the Fermi liquid (FL) laws in the clean limit, taking into account the quasiparticle damping. The latter yields a reduced value of the Lorenz number in the WiedemannFranz law. Our results indicate that the renormalization of the thermal conductivity and of the Seebeck coefficient can lead to a substantial enhancement of the electronic thermoelectric figure-ofmerit at low temperature.

Using the FL laws we discuss the low-temperature anomalies that show up in the electrical resistance of the intermetallic compounds with Cerium and Ytterbium ions, when studied as a function of pressure. Our calculations explain the sharp maximum of the coefficient of the $T^{2}$-term of the electrical resistance and the rapid variation of residual resistance found in a number of $\mathrm{Ce}$ and $\mathrm{Yb}$ intermetallics at some critical pressure.
\end{abstract}

PACS numbers: 75.30.Mb, 72.15.Jf, 62.50.+p, 75.30.Kz,

\section{INTRODUCTION}

The low-temperature charge and thermal transport of heavy fermions and valence fluctuators with $\mathrm{Ce}, \mathrm{Eu}, \mathrm{Yb}$ and $\mathrm{U}$ ions display interesting and complex behavior, like a striking correlation $\frac{1.2}{.}$ between the low-temperature Seebeck coefficient $\alpha(T)$ and the specific heat coefficient $\gamma=C_{V} / T$. In many of these systems the dimensionless ratio $q=|e| \lim _{T \rightarrow 0} \alpha / \gamma T$ is nearly the same, although the absolute values of $\gamma$ and $\alpha / T$ vary by orders of magnitude. In metallic systems, the data show ${ }^{3,4,5,6.7}$ small deviations from this universal behavior, due to the variation in carrier concentration, and in bad metals, $q$ can become quite large $\frac{1,2}{2}$. The Kadowaki-Woods (KW) ratio ${ }^{8}$ which is defined as $\rho(T) /(\gamma T)^{2}$, where $\rho(T)$ is the electrical resistivity, exhibits similar universal features, if one takes into account the effective low-temperature degeneracy of the $f$ multiplet, as defined by the multiplicity of the crystal field (CF) ground state ${ }^{9.10}$, the carrier concentration and the unit cell volume 11 .

The near constancy of the KW and $q$ ratios brings to the fore the validity of the Wiedemann-Franz (WF) law, $\kappa \rho / T=\mathcal{L}_{0}$, and a possibility of enhancing the electronic thermoelectric figure-of-merit in strongly correlated materials, $Z T=\alpha^{2} T / \kappa \rho$, where $\kappa$ is the electronic contribution to the thermal conductivity and $\mathcal{L}_{0}=\pi^{2} k_{B}^{2} / 3 e^{2}$ the Fermi liquid (FL) Lorenz number. When the WF law holds, metals must have a thermopower larger than $155 \mu \mathrm{V} / \mathrm{K}$ to achieve $Z T>1$; to date no metal has been found with so large a thermopower. In the temperature window where the effective Lorenz number $(\mathcal{L}=\kappa \rho / T)$ is reduced, one can achieve $Z T>1$ with substantially lower thermopowers, which might make it possible to find strongly correlated metals that can be used for cooling applications at low temperature.
The above-mentioned universality of the $\mathrm{KW}$ and $q$ ratios, typical of a Fermi liquid (FL) state, attracted considerable theoretical attention. Kontani ${ }^{9}$ explained the $\mathrm{KW}$ ratio using the orbitally degenerate periodic Anderson model that he solved by the quasiparticle (QP) approximation of Yosida and Yamada 12,13. Neglecting both the vertex corrections and the momentum dependence of the self-energy, he derived a $\mathrm{KW}$ ratio that depends on the degeneracy of the $f$ states and brings the experimental data closer to the universal (theoretical) curve $\underline{10}$. Miyake and Kohno $\frac{14}{14}$ calculated the $q$ ratio for the same QP dispersion as in Ref. 12, using an effective $N$-fold degenerate free-electron model in which the on-site correlation $U_{f f}$ is accounted for by the renormalized hybridization between the $c$ and $f$ states. Restricting the average number of $f$ electrons to $n_{f} \leq 1$, they treated the QPs as free fermions and assumed that the repeated impurity scattering gives rise to an energy dependent relaxation rate. Neglecting the QP damping due to Coulomb repulsion, Miyake and Kohno 14 calculated the low-temperature thermopower as a logarithmic derivative of the frequency-dependent conductivity 15 and show that the $q$ ratio is quasi-universal number.

The effect of electron-electron scattering on the transport coefficients has recently been studied by Grenzebach et al $\stackrel{16}{=}$, using the dynamical mean-field theory $\underline{17}$ (DMFT) of the periodic spin-1/2 Anderson model. The auxiliary impurity problem generated by the DMFT was solved by the numerical renormalization group (NRG) method, which discretizes the energy spectrum and defines the temperature as the difference between the two lowest energy states. This provides accurate results for the static properties at arbitrary temperature but cannot provide the thermal transport in the FL regime. The DMFT+NRG calculations indicate an enhancement of the figure of merit when the temperature is reduced be- 
low its value at the resistivity maximum, and show a breakdown of the WF law due to electron correlations. Recently, we calculated the thermopower and the $q$-ratio of the periodic Anderson model taking into account the $\mathrm{CF}$ splitting 18 and enforcing $n_{f} \leq 1$ at each lattice site (a realistic description of the $\mathrm{CF}$ states requires a local constraint). Solving the auxiliary impurity problem in the non-crossing approximation (NCA) we obtained a semi-quantitative description of the experimental data on heavy fermions and valence fluctuators in the incoherent regime. However, a detailed analysis of the DMT+NRG and the NCA results shows that nether method can describe the thermal transport much below the Kondo temperature $T_{K}$ nor establish the FL laws.

In this paper, we discuss the coherent thermal transport of the periodic Anderson model with $S U(N)$ symmetry in the limit of an infinitely large Coulomb repulsion between $f$ electrons. Such an effective $N$-fold degenerate model applies to intermetallic compounds with $\mathrm{Ce}$, $\mathrm{Eu}, \mathrm{Yb}$ and $\mathrm{U}$ ions in the FL regime, where the excited $\mathrm{CF}$ states can be neglected 19 . For a given compound, the number of effective channels depends on the pressure and doping. We assume that the model has been solved in thermal equilibrium, so that the FL scale $T_{0} \sim 1 / \gamma$ is known. The total particle number per unit cell $n(\mu)$ is also $n$ known; $\mu$ denotes the chemical potential. Alternatively, we can assume that the values of $\gamma$ and $n$ are taken from experiment. Using these equilibrium quantities, and enforcing the constraint $n_{f} \leq 1$ at each lattice site, we construct an analytic solution for the stationary heat and charge transport in the FL regime.

Unlike in previous work, we do not calculate the transport properties in the QP representation, because the operator algebra in the projected Hilbert space is not fermionic and the representation of the exact charge and heat current density operators is cumbersome. In the original fermionic representation, Mahan ${ }^{20}$ has demonstrated that the correlation functions between the above current density operators can be expressed in terms of transport integrals which differ only by powers of the excitation energy in their integrand, so that the same techniques can be used for their evaluation as in weakly correlated systems.

The transport coefficients are obtained by the DMFT and expressed in terms of the average conduction electron velocity, the renormalized density of conduction states, and the frequency and temperature dependent relaxation rate which explicitly takes into account the QP damping. Since we are considering the FL regime, we use the QP approximation of Yosida and Yamada 12 to relate all these quantities to the FL scale $T_{0}$ and to the unrenormalized density of $c$ states, $\mathcal{N}_{c}^{0}(\omega)$, evaluated at the shifted chemical potential $\mu_{L}=\mu+\Delta \mu$. The shift $\Delta \mu$ is determined by the Luttinger theorem. Using the Sommerfeld expansion, we obtain the universal FL laws which show that the KW ratio depends not only on the multiplicity of the $f$ state and the average FS velocity, but also on the carrier concentration and the unit cell volume, as observed experimentally 11 . As regards the $q$-ratio, we find that changes in the carrier concentration, induced by pressure or chemical pressure, lead to deviations from universality. We also find large deviations from the WF law due to the lowering of the effective Lorenz number, which can lead to a substantial enhancement of the thermoelectric figure-of-merit $Z T>1$. The change in the effective degeneracy of the model induced by pressure explains the pronounced maximum of the coefficient of the $T^{2}$ term of the electrical resistance $\frac{21,22,23,24}{2}$ and the rapid variation of residual resistance $\frac{21,22}{}$, found in a number of Ce and $\mathrm{Yb}$ intermetallics at some critical pressure.

The usefulness of the approximate analytic solution of the DMFT equations becomes apparent when we realize that a numerical calculation of the above mentioned universal ratios encounters serious difficulty, and neither the NRG, nor exact diagonalization, nor quantum Monte Carlo approaches provide accurate transport coefficients in the FL regime (especially when the coherence temperature is low). Combining analytical and numerical results enables a reliable estimate of transport coefficients at arbitrary temperatures, which is needed if the model is to be compared with experimental data.

The rest of this paper is organized as follows: Sec. II describes the DMFT calculations of transport coefficients in the low-temperature limit, in Sec. III we use the QP approximation to calculate the renormalized density of states and transport relaxation time in the FL regime, Sec. IV establishes the FL laws, in Sec. V we use our results to discuss the experimental data, and Sec. VI provides the summary and conclusions.

\section{FORMALISM FOR THE TRANSPORT COEFFICIENTS}

The $S U(N)$-symmetric periodic Anderson model is written in the standard form 19 ,

$$
\mathcal{H}=\mathcal{H}_{\text {band }}+\mathcal{H}_{\text {imp }}+\mathcal{H}_{\text {mix }}-\mu \mathcal{N} .
$$

Here, $\mathcal{H}_{\text {band }}$ describes the conduction $(c)$ band with unperturbed dispersion $\epsilon_{\mathbf{k}}$, assumed to be a even function of k. Because we have an $S U(N)$ symmetric model, there are $N$ distinct flavors of conduction electrons, which are labeled by index $\sigma$, so that

$$
\mathcal{H}_{\text {band }}=\sum_{\sigma=1}^{N} \sum_{\mathbf{k}} \epsilon_{\mathbf{k}} c_{\mathbf{k} \sigma}^{\dagger} c_{\mathbf{k} \sigma} .
$$

The operators $c_{\mathbf{k} \sigma}^{\dagger}\left(c_{\mathbf{k} \sigma}\right)$ create (annihilate) a band electron with momentum $\mathbf{k}$ and flavor $\sigma$. The non-interacting density of conduction states $\mathcal{N}_{c}^{0}(\omega)$ is calculated for each flavor and any $\epsilon_{\mathbf{k}}$ as $\mathcal{N}_{c}^{0}(\omega)=1 /\left(\mathcal{N}_{i} \mathcal{V}\right) \sum_{\mathbf{k}} \delta\left(\omega-\epsilon_{\mathbf{k}}\right)$, where $\mathcal{N}_{i}$ is the number of lattice sites and $\mathcal{V}$ the volume of the primitive unit cell. The characteristic bandwidth of the unperturbed $c$-DOS is $W$. The number of conduction electrons is $N n_{c}$ (per unit cell), i.e., there are 
$n_{c}$ conduction electrons of each flavor. We assume an infinite Coulomb repulsion, $U_{f f} \rightarrow \infty$, and describe the localized $4 f$ states by $\mathcal{H}_{i m p}$,

$$
\mathcal{H}_{i m p}=\sum_{\sigma=1}^{N} \sum_{i} E_{f}^{\sigma} \mathcal{P} f_{i \sigma}^{\dagger} f_{i \sigma} \mathcal{P},
$$

where $E_{f}^{\sigma}$ labels the site energy of flavor $\sigma$ and $\mathcal{P}$ projects onto the subspace with zero or one total $f$-electrons per site. The number of $f$ electrons is restricted to $n_{f} \leq 1$, i.e., there are $n_{f} / N$ localized electrons per flavor. The hybridization between same flavor $c$ and $f$ electrons is described by $\mathcal{H}_{m i x}$

$$
\mathcal{H}_{\text {mix }}=\sum_{\sigma=1}^{N} \sum_{i, j} V_{i j}^{\sigma} \mathcal{P}\left(f_{i \sigma}^{\dagger} c_{i \sigma}+c_{i \sigma}^{\dagger} f_{i \sigma}\right) \mathcal{P},
$$

where the conduction-electron operators are now written in real space. The total electron number operator is $\mathcal{N}=$ $\sum_{\sigma} \sum_{i}\left(c_{i \sigma}^{\dagger} c_{i \sigma}+f_{i \sigma}^{\dagger} f_{i \sigma}\right)$ and the chemical potential $\mu$ is adjusted to keep the total particle number $n=N n_{c}+n_{f}$ constant (as a function of temperature or pressure). For a degenerate paramagnetic state, all flavors are equivalent and the label $\sigma$ can be dropped.

The intermetallic compounds with $4 f$ ions are described by the $N$-fold degenerate model with on-site hybridization, $V_{i j}=V \delta_{i j}$, which simplifies the calculations. For large $N$, an exact solution is provided by the meanfield or the slave boson solution 19 but for small $N$ the correlations give rise to the Kondo effect which cannot be described by mean field theories. The physically relevant value of $N$ depends on the effective degeneracy of the $4 f$ state. A single electron (or hole) in the lowest spin-orbit state of the $4 f$ shell of $\mathrm{Ce}(\mathrm{Yb})$ is $2 J+1$-fold degenerate, where $J=5 / 2$ for Ce and $J=7 / 2$ for $\mathrm{Yb}$. This degeneracy is further reduced by $\mathrm{CF}$ splitting and for small hybridization, and temperatures at which the excited CF states are unoccupied, the value of $N$ is determined by the degeneracy of the lowest $\mathrm{CF}$ state. If the hybridization is such that the CF splitting does not occur, or the excited states are thermally occupied, the effective degeneracy is defined by the lowest spin-orbit multiplet, which is a sextet for Ce and an octet for $\mathrm{Yb}$. In all the physically relevant cases, $2 \leq N \leq 8$, the system exhibits the Kondo effect.

At high temperatures, the qualitative solution can be obtained by perturbative scaling 19 which shows that the properties depend on an exponentially small energy scale $T_{K} \simeq W \exp (-1 / N g)$, where $g$ is the dimensionless coupling constant $g=V^{2} \mathcal{N}_{c}^{0}(\mu) / E_{f}$. At temperature $T_{K}$ the perturbation theory breaks down. The low-temperature thermodynamic quantities of the $S U(N)$ model are given very accurately by the DMFT+NRG and we assume that we know the numerical value of Fermi liquid scale $T_{0}=\pi^{2} k_{B}^{2} / 3 \gamma \mathcal{V}$, where the linear coefficient of the lowtemperature specific heat, $\gamma$, is measured per unit volume. Alternatively, $T_{0}$ can be estimated from the slave boson solution or taken from experiment. Unlike the situation for the static thermodynamic quantities, no currently available numerical method provides a reliable solution for the transport coefficients at low temperatures. In this paper, we evaluate the heat and charge transport of the $S U(N)$ symmetric Anderson model by the Fermi liquid theory $\underline{12}$ which holds for $T \leq T_{0}$.

For the static and uniform transport we need the $\mathbf{q} \rightarrow 0$ component of the charge and heat current density operators which are obtained by commuting the Hamiltonian with the appropriate polarization operators ${ }^{25}$. The total current density obtained in such a way satisfies

$$
\mathbf{j}_{c}=\lim _{\tau, \tau^{\prime} \rightarrow 0} \frac{e N}{\mathcal{V N} \sum_{i}} \sum_{\mathbf{k}} \mathbf{v}_{\mathbf{k}} c_{\mathbf{k} \sigma}^{\dagger}(\tau) c_{\mathbf{k} \sigma}\left(\tau^{\prime}\right),
$$

where $\mathbf{v}_{\mathbf{k}}=\nabla \epsilon_{\mathbf{k}} / \hbar$ is the band velocity of the noninteracting (and unhybridized) $c$-states. For constant hybridization the energy current density is given by a similar expression which shows that the model satisfies the Jonson-Mahan theorem ${ }^{20}$. This allows us to express the charge conductivity by $\sigma(T)=e^{2} N L_{11}$, the thermopower by $\alpha(T)|e| T=-L_{12} / L_{11}$, and the electronic contribution to the thermal conductivity by $\kappa(T) T=$ $N\left(L_{22}-L_{12}^{2} / L_{11}\right)$. In each of these expressions we have introduced the (single-flavor) transport integrals:

$$
L_{m n}=\int d \omega\left(-\frac{d f}{d \omega}\right) \omega^{m+n-2} \Lambda(\omega, T) .
$$

where $f(\omega)=1 /[1+\exp (\beta \omega)]$ is the Fermi-Dirac distribution function, $\omega$ is measured with respect to the chemical potential $\mu$, and $\Lambda(\omega, T)$ is a function calculated in Appendix $\mathrm{A}$ by the Kubo linear response theory.

At low temperature $(-d f / d \omega)$ approaches a delta function and the main contribution to the integrals in Eq. (6) comes from the low-energy excitations within the Fermi window, $|\omega| \lesssim k_{B} T$, and with the wave vectors in the vicinity of the Fermi surface. In the Fermi liquid state $\Lambda(\omega, T)$ can be calculated in a straightforward way (for details see Appendix $\mathrm{A}$ ) which yields in the $\omega, T \rightarrow 0$ limit

$$
\Lambda(\omega, T)=\frac{1}{3} v_{F}^{2} \mathcal{N}_{c}(\omega) \tau(\omega, T),
$$

for a three-dimensional system. Here, $v_{F}^{2}$ denotes the average of $v_{\mathbf{k}}^{2}$ over the renormalized Fermi surface of hybridized states, $\tau(\omega, T)$ is the transport relaxation time, given by the momentum-independent self-energy of the $c$ electrons,

$$
\tau(\omega, T)=\frac{-\hbar}{\operatorname{Im} \Sigma_{c}\left(\omega^{+}, T\right)},
$$

and $\mathcal{N}_{c}(\omega)$ is the renormalized DOS of $c$ electrons (for a single flavor),

$$
\mathcal{N}_{c}(\omega)=\frac{1}{\mathcal{V} \mathcal{N}_{i}} \sum_{\mathbf{k}} A_{c}(\mathbf{k}, \omega)
$$


The spectral function of the $c$ electrons

$$
A_{c}(\mathbf{k}, \omega)=-\frac{1}{\pi} \operatorname{Im} G_{c}\left(\mathbf{k}, \omega^{+}\right)
$$

is obtained by evaluating the retarded Green's function $G_{c}(\mathbf{k}, z)$ just above the real axis, where $z=\omega^{+}=\omega+i \delta$ and $\delta \rightarrow 0$ from positive values.

The Green's functions of the periodic Anderson model are defined by the time-ordered thermal averages in the usual way and are cast in the Dyson form using the equation of motion or the diagrammatic expansion. The Dyson equations for $c$ and $f$ Green's function read ${ }^{12,13}$

$$
G_{c}(\mathbf{k}, z)=\frac{z-E_{f}-\Sigma_{f}(z)+\mu}{\left[z-\epsilon_{\mathbf{k}}+\mu\right]\left[z-E_{f}-\Sigma_{f}(z)+\mu\right]-V^{2}}
$$

and

$$
G_{f}(\mathbf{k}, z)=\frac{z-\epsilon_{\mathbf{k}}+\mu}{\left[z-\epsilon_{\mathbf{k}}+\mu\right]\left[z-E_{f}-\Sigma_{f}(z)+\mu\right]-V^{2}},
$$

where $z$ denotes a variable in the complex energy plane and $\Sigma_{f}(z)$ the local self-energy of $f$ electrons which describes the renormalization of the (hybridized) $f$ states due to local Coulomb interaction. The Dyson equation for the conduction electrons can also be written in the alternative form

$$
G_{c}(\mathbf{k}, z)=\frac{1}{z-\epsilon_{\mathbf{k}}+\mu-\Sigma_{c}(z)}
$$

where the local self-energy of the conduction electrons satisfies

$$
\Sigma_{c}(z)=\frac{V^{2}}{z-E_{f}+\mu-\Sigma_{f}(z)}
$$

This self-energy describes the renormalization of the unhybridized $c$ states due to the scattering on the $f$ states, i.e., $\Sigma_{c}$ includes the hybridization and the correlation effects. The DMFT condition ensures $\Sigma_{f}\left(\omega^{+}, T\right)$ to be local but the locality of $\Sigma_{c}\left(\omega^{+}, T\right)$ also requires the hybridization to be momentum independent. The dc transport relaxation time is obtained by substituting $\Sigma_{c}\left(\omega^{+}, T\right)$ into Eq. (8) which yields

$$
\frac{\tau(\omega, T)}{\hbar} \simeq \frac{\left(\omega-E_{f}-\operatorname{Re} \Sigma_{f}+\mu\right)^{2}+\left(\delta-\operatorname{I} m \Sigma_{f}\right)^{2}}{V^{2}\left(\delta-\operatorname{I} m \Sigma_{f}\right)}
$$

where the $T, \omega$-dependence is due to $\Sigma_{f}(\omega, T)$. To account for the residual impurity scattering, which is present in any sample, we assume that $\delta$ in Eq. (15) has a small but finite value. For temperatures such that $\left|\operatorname{Im} \Sigma_{f}(\omega, T)\right| \gg \delta$ this additional scattering is neglected and we discuss the temperature dependence of transport coefficients by setting $\delta=0$. At $\mathrm{T}=0$ the self energy vanishes and $\tau(\omega, T)$ becomes a constant. The pressure dependence of the residual resistance is discussed using Eq. (7) with a constant relaxation time, which neglects the renormalization effects due to the repeated scattering on the impurities. Where appropriate, the clean limit implies we are in the regime where $\delta \ll\left|\operatorname{Im} \Sigma_{f}(\omega, T)\right|$, whereas, we must examine the dirty limit, where $\delta>\left|\operatorname{Im} \Sigma_{f}(\omega, T)\right|$, to describe residual resitance data.

\section{THE FERMI LIQUID APPROACH}

In the FL regime, the imaginary part of $\Sigma_{f}$ is small and can be neglected when discussing the renormalized excitation spectrum in the limit $T, \omega \rightarrow 0$. Expanding $\Sigma_{f}(\omega)$ through linear order in frequency we find in that limit

$$
\omega-\left[E_{f}+\operatorname{Re} \Sigma_{f}(\omega)-\mu\right] \approx\left(\omega-\tilde{\omega}_{f}\right) Z_{f}^{-1}+\mathcal{O}\left(\omega^{2}\right)
$$

with $Z_{f}^{-1}=\left[1-\partial \Sigma_{f} /\left.\partial \omega\right|_{\omega=0}\right]$ being the enhancement factor $\left(0 \leq Z_{f} \leq 1\right)$ and $\tilde{\omega}_{f}=\left[E_{f}+\operatorname{Re} \Sigma_{f}(0)-\mu\right] Z_{f}$. The parameters $\tilde{\omega}_{f}$ and $Z_{f}$ appear in the the description of the model at low-temperatures.

If we ignore the imaginary part of the self-energy, both Green's functions in Eqs. (11) and (12) develop poles 12 which define the QP excitations with wave vector $\mathbf{k}$. Using Eq. (16) we can write the secular equation for the QP excitations in terms of the renormalized quantities, as

$$
\left(\omega-\epsilon_{\mathbf{k}}+\mu\right)\left(\omega-\tilde{\omega}_{f}\right)-\tilde{V}^{2}=0
$$

with $\tilde{V}=V \sqrt{Z_{f}}$ the renormalized hybridization. The roots $\omega=\Omega_{\mathbf{k}}^{ \pm}$describe two QP branches

$$
\Omega_{\mathbf{k}}^{ \pm}=\frac{1}{2}\left[\left(\epsilon_{\mathbf{k}}-\mu+\tilde{\omega}_{f}\right) \pm \sqrt{\left(\epsilon_{\mathbf{k}}-\mu-\tilde{\omega}_{f}\right)^{2}+4 \tilde{V}^{2}}\right]
$$

separated by the hybridization gap $2 \tilde{V}$. These excitations are only defined for energies close to the chemical potential, where $\mathrm{I} m \Sigma_{f}$ can be neglected. For large $\omega$ the QP approximation breaks down and Eq. (17) is unphysical. At the FS we have $\Omega_{\mathbf{k}_{F}}^{-}=0$ or $\Omega_{\mathbf{k}_{F}}^{+}=0$, depending on the value of $N n_{c}+n_{f}$. For Ce and Eu systems we place the bare $f$ level below the chemical potential, which puts $\mu$ close to the top of the lower QP branch. From $\Omega_{\mathbf{k}_{F}}^{-}=0$ we see that $\mathbf{k}_{F}$ is then close to the zone boundary, where $\epsilon_{\mathbf{k}_{F}}-\mu \simeq W$ ( $W$ denotes the half-width of the unperturbed $c$ band). For $\mathrm{Yb}$ compounds, the bare level is above $\mu$, so that the lower QP branch is full and the chemical potential is close to the bottom of the upper branch. The equation $\Omega_{\mathbf{k}_{F}}^{+}=0$ gives $\mathbf{k}_{F}$ close to the zone center, such that $\epsilon_{\mathbf{k}_{F}}-\mu \simeq-W$. Close to the FS, the $\mathrm{QP}$ dispersion is very weak and $\Omega_{\mathbf{k}}^{ \pm}$describes two heavy QP bands with a half-width equal to $\tilde{V}_{f}^{2} / W$.

The above derivation treats the QP as a noninteracting Fermi gas with effective parameters $\tilde{\omega}_{f}$ and $Z_{f}$ (or $\left.\tilde{V}\right)$. These parameters can be related to the linear coefficient of the specific heat, which is assumed to 
be known. Using the expression for total QP density of states

$$
\mathcal{N}^{Q P}(\omega)=\frac{1}{\mathcal{V} \mathcal{N}_{i}} \sum_{\mathbf{k}} \delta\left(\omega-\Omega_{\mathbf{k}}^{ \pm}\right)
$$

and the fact that the QP excitations defined by Eq. (17) are infinitely long-lived $\left(\operatorname{Im} \Sigma_{f}(\omega)=0\right.$ at $\left.T=0\right)$ we write

$$
\gamma=\frac{\pi^{2} k_{B}^{2}}{6} N \mathcal{N}^{Q P}(0)=\frac{\pi^{2} k_{B}^{2}}{3 \mathcal{V}} \frac{1}{k_{B} T_{0}},
$$

where the $N$-fold degeneracy of the system has been taken into account. The relationship between $\gamma$ and $\mathcal{N}^{Q P}$ is derived assuming that thermally excitated QPs increase the average energy per unit volume by approximately $\Delta E \simeq\left(k_{B} T\right)^{2} N \mathcal{N}^{Q P}(0)$. The FL parameter $T_{0}$ introduced in Eq.(20) defines the low-temperature scaling behavior of all physical quantities. In many systems, it also sets the temperature at which the QP description breaks down. This temperature might or might not coincide with the high-temperature Kondo scale of the system. By definition, the inverse of $T_{0}$ is given by the specific heat coefficient or, equivalently, by the density of the QP excitations of the $S U(N)$ model, which can be calculated very accurately in thermal equilibrium. As shown below, the low-temperature thermal transport is also characterized by $T_{0}$.

The FL scale $T_{0}$ can be related to the partial densities of $f$ and $c$ states 12 by expanding Eqs. (11) and (12) for small $\omega$. This yields the spectral functions,

$$
A_{c}(\mathbf{k}, \omega) \approx a_{\mathbf{k}}^{c}(\omega) \delta\left(\omega-\Omega_{\mathbf{k}}^{ \pm}\right)
$$

and

$$
A_{f}(\mathbf{k}, \omega) \approx a_{\mathbf{k}}^{f}(\omega) \delta\left(\omega-\Omega_{\mathbf{k}}^{ \pm}\right)
$$

where we used the fact that $\omega=\Omega_{\mathbf{k}}^{ \pm}$can only be satisfied with one of the roots, and introduced the coefficients

$$
a_{\mathbf{k}}^{c}(\omega)=\left[1+\frac{\tilde{V}^{2}}{\left(\omega-\tilde{\omega}_{f}\right)^{2}}\right]^{-1}
$$

and

$$
a_{\mathbf{k}}^{f}(\omega)=Z_{f} \frac{\tilde{V}^{2}}{\left(\omega-\tilde{\omega}_{f}\right)^{2}} a_{\mathbf{k}}^{c}(\omega) .
$$

These coefficients satisfy the FL sum rule ${ }^{12}$,

$$
a_{\mathbf{k}}^{c}(\omega)+Z_{f}^{-1} a_{\mathbf{k}}^{f}(\omega)=1 .
$$

When we substitute Eq. (25) into Eq. (19) and use Eq. (20), the summation of $A_{c}(\mathbf{k}, \omega)$ and $A_{f}(\mathbf{k}, \omega)$ over the first Brillouin zone yields

$$
\frac{2}{\mathcal{V}} \frac{1}{N k_{B} T_{0}}=\mathcal{N}_{c}(0)+Z_{f}^{-1} \mathcal{N}_{f}(0),
$$

which shows that the FL scale is set by the product of the large enhancement factor $1 / Z_{f}$ and $\mathcal{N}_{f}(0)$.

To relate $T_{0}$ to the renormalized parameters $\tilde{\omega}_{f}$ and $Z_{f}$, we express, first, the renormalized $c$ and $f$ DOS in terms of the density of unhybridized band states. Using the identity $\delta\left(\omega-\Omega_{\mathbf{k}}^{ \pm}\right)=\delta\left(\epsilon_{\mathbf{k}}-\epsilon_{\mathbf{k} \omega}^{ \pm}\right) /\left|d \Omega_{\mathbf{k}}^{ \pm} / d \epsilon_{\mathbf{k}}\right|$ and $d \Omega_{\mathbf{k}}^{ \pm} / d \epsilon_{\mathbf{k}}=\left.a_{\mathbf{k}}^{c}(\omega)\right|_{\omega=\Omega_{\mathbf{k}}^{ \pm}}$, where $\epsilon_{\mathbf{k}_{\omega}}^{ \pm}$is the solution of Eq. (17) for a given (small) $\omega$, we obtain from Eqs. (21) and (22) the result

$$
\mathcal{N}_{c}(\omega)=\mathcal{N}_{c}^{0}\left(\omega+\mu-\frac{\tilde{V}^{2}}{\omega-\tilde{\omega}_{f}}\right),
$$

and

$$
\mathcal{N}_{f}(\omega)=\frac{Z_{f} \tilde{V}^{2}}{\left[\omega-\tilde{\omega}_{f}\right]^{2}} \mathcal{N}_{c}(\omega)
$$

The expressions in Eqs. (27) and (28) are similar in spirit and in form to those in Refs. 26 and 27, where the fully interacting DOS of the periodic Anderson model is equated to the DOS of a non-interacting $U=0$ model. The Green's functions of that model are defined by Eqs. (11) and (12) for $\Sigma_{f}(\omega)=0$. Here, we find it more convenient to relate $\mathcal{N}_{c}(\omega)$ to the DOS of an unhybridized conduction band which is obtained from Eq. (13) for $\Sigma_{c}(\omega)=0$. At low temperatures, where only the hybridized bands have physical relevance, this auxiliary conduction band is just a convenient mathematical construct. But at high temperatures, where the model has to account for the scattering of conduction states on localized paramagnetic $f$ states, the unhybridized conduction band is physically relevant.

The auxiliary model provides the renormalized Fermi surface (FS) of the periodic Anderson model simply by the Luttinger theorem, i.e., from the fundamental Fermi liquid relation which states that the volume of the FS cannot be changed by interactions. Using the standard form of the $c$ electron Green's function [in Eq. (13)] and the fact that $\Sigma_{c}(z)$ is momentum independent (this holds for momentum-independent hybridization in infinite dimensions) we immediately learn that the FS of the auxiliary band model (with $n=N n_{c}+n_{f}$ electrons per unit cell) coincides with the FS of the periodic Anderson model with the same number of electrons per cell. The shape of the renormalized FS is obtained by solving Eq. (17) at $\omega=0$, which yields the implicit equation

$$
\epsilon_{\mathbf{k}_{F}}=\mu+\frac{\tilde{V}^{2}}{\tilde{\omega}_{f}}
$$

in terms of the non-interacting dispersion. We recall that $\mu$ is fixed by the condition

$$
n=N n_{c}+n_{f}=N \mathcal{V} \int_{-\infty}^{\mu} d \omega\left[\mathcal{N}_{c}(\omega)+\mathcal{N}_{f}(\omega)\right]
$$

The auxiliary model has exactly the same FS, determined by the equation

$$
\epsilon_{\mathbf{k}_{F}}=\mu_{L}
$$


where $\mu_{L}$ is obtained from the integral

$$
n=N \mathcal{V} \int_{-\infty}^{\mu_{L}} d \omega \mathcal{N}_{c}^{0}(\omega)
$$

i.e., $\mu_{L}$ is the chemical potential of a conduction band with $n=n_{c}+n_{f}$ electrons. The same chemical potential $\mu\left(\mu_{L}\right)$ appears in Eqs. (29) and (30) [Eqs. (31) and (32)], because Luttinger's theorem ensures that the number of k-points enclosed by the FS coincides with the total number of electrons in the system. Equations (29) and (31) give the shift

$$
\Delta \mu=\mu_{L}-\mu=\frac{\tilde{V}^{2}}{\tilde{\omega}_{f}} .
$$

Substituting Eqs. (27) and (28) into Eq. (26) and using Eq. (33) to eliminate $\tilde{V}^{2} / \tilde{\omega}_{f}$, we find the relationship between $\tilde{\omega}_{f}$ and $T_{0}$,

$\tilde{\omega}_{f}=\Delta \mu \frac{N \mathcal{N}_{c}^{0}\left(\mu_{L}\right) \mathcal{V} k_{B} T_{0} / 2}{1-N \mathcal{N}_{c}^{0}\left(\mu_{L}\right) \mathcal{V} k_{B} T_{0} / 2} \simeq \frac{N}{2} \Delta \mu \mathcal{N}_{c}^{0}\left(\mu_{L}\right) \mathcal{V} k_{B} T_{0}$,

where the last expression neglects the exponentially small term in the denominator. The sign of $\tilde{\omega}_{f}$ is set by $\Delta \mu$ which is positive for $\mathrm{Ce}$ and $\mathrm{Eu}$ compounds and negative for $\mathrm{Yb}$ compounds. The enhancement factor is obtained from Eq. (33) as $Z_{f}=\Delta \mu \tilde{\omega}_{f} / V^{2}$.

The ratio $2 \tilde{\omega}_{f} / T_{0}=\Delta \mu \mathcal{V} \mathcal{N}_{c}^{0}\left(\mu_{L}\right)$ depends, for a given $n(\mu)$, on the renormalized chemical potential $\mu$, the auxiliary one $\mu_{L}$, and the unit cell volume $\mathcal{V}$. While $\mu_{L}$ is easily obtained from $n(\mu)$, the value of $\mu$ is difficult to find without numerical calculations. Except, for very small $\tilde{V}$, large $N$, and for $n_{f} \simeq 1$ and $n_{c} \simeq 1 / 2$, when each conduction band is close to half-filling. In that case, the renormalization does not change much the partial occupancies of the $f$ and $c$ states, and we can approximate $n_{c} \simeq \mathcal{V} \int_{-\infty}^{\mu} d \omega \mathcal{N}_{c}^{0}(\omega)$, which yields

$$
N \mathcal{V} \int_{\mu}^{\mu_{L}} d \omega \mathcal{N}_{c}^{0}(\omega) \simeq n-N n_{c}=n_{f}
$$

where we have implicitly assumed $\mu_{L}>\mu$, which applies to Ce and Eu compounds. A similar result can be obtained for $\mathrm{Yb}$ compounds after an electron-hole transformation. For large $N$ the integral is small and since the integrand has a maximum around $\mu$ (the auxiliary band is close to half-filling), it follows that $\Delta \mu$ is small. Using $\mathcal{N}_{c}^{0}\left(\mu_{L}\right) \simeq \mathcal{N}_{c}^{0}(\mu)$ we estimate

$$
\mathcal{V} \int_{\mu}^{\mu_{L}} d \omega \mathcal{N}_{c}^{0}(\omega) \simeq \Delta \mu \mathcal{V} \mathcal{N}_{c}^{0}\left(\mu_{L}\right) \simeq \frac{n_{f}}{N} .
$$

Thus, for large $N$ and $n_{f} \simeq 1$, we have a simple relation $\tilde{\omega}_{f} \simeq n_{f} k_{B} T_{0} / 2$. For small $N$, and/or small $n_{f}$, Eq. (35) still holds but we cannot claim that $\Delta \mu$ is small. If $\mathcal{N}_{c}^{0}(\omega)$ decreases rapidly for $\omega>\mu$, as it usually does, we can conclude $\Delta \mu \mathcal{V} \mathcal{N}_{c}^{0}\left(\mu_{L}\right) \ll n_{f} / N \ll \Delta \mu \mathcal{V} \mathcal{N}_{c}^{0}(\mu)$ but cannot express $\Delta \mu \mathcal{V} \mathcal{N}_{c}^{0}\left(\mu_{L}\right)$ in terms of $n_{f}$ or relate $\tilde{\omega}_{f}$ to $T_{0}$ in a simple way, as in the large- $N$ limit.
The auxiliary model with unhybridized conduction electrons has a simple physical interpretation for systems with small hybridization and $n_{f} \simeq 1$, i.e., for heavy fermions with very low Kondo temperature. In such systems the $4 f$ ions are in a well defined valence state, the low-energy dynamics is dominated by spin fluctuations, and $n_{f}$ is temperature-independent ${ }^{19}$. At low temperatures, the renormalized FS is defined by the set of $\mathbf{k}$ vectors satisfying $\epsilon_{\mathbf{k}}=\mu+\Delta \mu$. This FS is large, because it encloses $\left(n_{c}+n_{f} / N\right)$ states of each flavor. At high temperatures, the Fermi surface is small, because it encloses only $n_{c}$ conduction states ( $f$ states are localized and do not contribute to the Fermi volume). Ignoring the fact that thermal fluctuations remove the discontinuity in the Fermi distribution function, we approximate the renormalized FS by the FS of unhybridized conduction states. (This holds for $T \ll W$.) On the other hand, the condition $\epsilon_{\mathbf{k}}=\mu$ defines a set of $\mathbf{k}$ vectors which are close to the FS of the auxiliary band with $n_{c}$ electrons. This FS is also small, because it encloses only $n_{c}$ states of each flavor. Since $n_{f}$ and $n_{c}$ are temperature-independent, the shift $\Delta \mu$ provides the difference between the FS of the high-temperature paramagnetic phase with $N n_{c}$ conduction electrons (and $n_{f}$ localized states), and the FS of the low-temperature FL phase with $N$ bands containing each $n_{c}+n_{f} / N$ hybridized states. The above considerations show that the formation of the coherent QP bands is accompanied by a 'jump' of the Fermi volume from a small to a large value, and that the large FS encloses $n_{f} / N$ additional states. The auxiliary model approximates the conduction band of the periodic Anderson model and allows us to estimate this jump.

So far, we neglected the imaginary part of self-energy, since we considered only the low-energy excitations at $T=0$ In order to calculate the transport properties at low but nonzero temperature, we have to include the QP damping which is given by the imaginary part of the self energy (in the clean limit). To estimate $\operatorname{Im} \Sigma_{f}(\omega, T)$ at low temperatures, we use the diagrammatic analysis of Yosida and Yamada ${ }^{12}$. In infinite dimensions, the expansion is in terms of the local Green's functions, and produces the FL expression ${ }^{9.12}$

$$
-\operatorname{Im} \Sigma_{f}(\omega, T) \simeq \frac{\pi}{2}\left[\omega^{2}+\left(\pi k_{B} T\right)^{2}\right](N-1)\left[\mathcal{V} \mathcal{N}_{f}(0)\right]^{3} \Gamma_{f}^{2},
$$

where $\Gamma_{f}$ is the irreducible 4-point scattering vertex for electrons with different flavors. Eq. (37) is a straightforward generalization of the result produced by second order perturbation theory in which the bare interaction $U_{f f}$ is replaced by the scattering vertex $\Gamma_{f}$. In the limit of large correlations, when the charge fluctuations are suppressed, the Ward identity 12,13 gives $Z_{f}^{-1}=(N-1) \mathcal{V} \mathcal{N}_{f}(0) \Gamma_{f}$, such that $\gamma=\left(\pi^{2} k_{B}^{2} / 6\right) N(N-$ 1) $\mathcal{V}\left[\mathcal{N}_{f}(0)\right]^{2} \Gamma_{f}$, where we neglect the much smaller conduction electron contribution to $\gamma$. Substituting into 
Eq. (37) yields in the $T, \omega \rightarrow 0$ limit $\underline{\underline{9}}$

$$
\operatorname{Im} \Sigma_{f}(\omega, T) \simeq-2 \pi \frac{\omega^{2}+\left(\pi k_{B} T\right)^{2}}{(N-1) N^{2} \mathcal{V} \mathcal{N}_{f}(0)\left(k_{B} T_{0}\right)^{2}}
$$

Expanding $\Sigma_{c}(\omega, T)$ in Eq. (14) into a power series [with the real and imaginary part of $\Sigma_{f}(\omega, T)$ given by Eqs. (16) and (38), respectively] we obtain the self energy of $c$ electrons in the FL form. That is, in the FL regime, the real part of $\Sigma_{c}(\omega, T)$ is a constant and the imaginary part is a quadratic function of $T$ and $\omega$.

Inserting $\Sigma_{f}(\omega, T)$ in Eq. (15) for $\tau(\omega, T)$ and using $Z_{f} \tilde{V}^{2} / \mathcal{N}_{f}(0)=\tilde{\omega}_{f}^{2} / \mathcal{N}_{c}(0)$ yields for $T, \omega \rightarrow 0$ the leading term

$$
\begin{aligned}
\tau(\omega, T) & =\frac{\hbar(N-1) N^{2} \mathcal{V} \mathcal{N}_{c}^{0}\left(\mu_{L}\right) k_{B}^{2} T_{0}^{2}}{2 \pi \tilde{\omega}_{f}^{2}} \frac{\left(\omega-\tilde{\omega}_{f}\right)^{2}}{\left(\pi k_{B} T\right)^{2}+\omega^{2}} \\
& \simeq \tau_{0}(T)\left(1-\frac{\omega}{\tilde{\omega}_{f}}\right)^{2}\left(1-\frac{\omega^{2}}{\pi^{2} k_{B}^{2} T^{2}}\right)
\end{aligned}
$$

where

$$
\tau_{0}(T)=\frac{\hbar(N-1) N^{2} \mathcal{V} \mathcal{N}_{c}^{0}\left(\mu_{L}\right)}{2 \pi^{3}} \frac{T_{0}^{2}}{T^{2}} .
$$

The second line in Eq. (39) emphasizes the fact that the limit $\omega \rightarrow 0$ is taken before $T \rightarrow 0$ and that $\Sigma_{f}\left(\omega^{+}, T\right)$ is only known up to the $\omega^{2}$ terms, so that the Sommerfeld expansion cannot be extended beyond the second order (up to that order both forms produce the same result). The first (second) bracket in Eq. (39) is due to the real (imaginary) part of $\Sigma_{f}\left(\omega^{+}, T\right)$. Unlike $\Sigma_{c}(\omega, T)$, the FL laws produced by the Sommerfeld expansion of transport coefficients are affected not only by the $\omega$-dependence of $\operatorname{Im} \Sigma_{f}(\omega, T)$ but of Re $\Sigma_{f}(\omega, T)$ as well.

In order to make an estimate of the slope of the renormalized $f$ DOS, which is needed for thermal transport, we invoke the DMFT condition. This condition also provides a physical interpretation of the low-energy parameter $\tilde{\omega}_{f}$. In DMFT, we compute the local Green's function from the local self-energy

$$
\begin{aligned}
G_{f}(z) & =\sum_{\mathbf{k}} G_{f}(\mathbf{k}, z) \\
& =\mathcal{V} \int d \epsilon \mathcal{N}_{c}^{0}(\epsilon) \frac{1}{z-E_{f}+\mu-\Sigma_{f}(z)-\frac{V^{2}}{z-\epsilon+\mu}},
\end{aligned}
$$

map it onto the Green's function of an effective single impurity Anderson model with a hybridization function $\Delta(z)$

$$
F(z)=\frac{1}{z-E_{f}+\mu-\Delta(z)-\Sigma_{f}(z)},
$$

and adjust $\Delta(z)$ to make $G_{f}(z)$ and $F(z)$ identical. The DMFT procedure works because the large-dimensional limit guarantees that the functional relationship between the local self-energy and the local Green's function for the lattice is identical to the functional relationship between the self-energy for the impurity and the impurity Green's function. In the limit where $T=0$ and $\omega \rightarrow 0$, we approximate $\Delta(\omega)=i \Delta_{0}$ with the constant $\Delta_{0}<0$ and write the DMFT condition at low frequencies as

$$
\mathcal{N}_{f}(\omega)=-\frac{1}{\pi} \operatorname{Im} F\left(\omega^{+}\right) \simeq \frac{1}{\pi\left|\Delta_{0}\right|} \frac{\tilde{\Delta}_{f}^{2}}{\left(\omega-\tilde{\omega}_{f}\right)^{2}+\tilde{\Delta}_{f}^{2}},
$$

where $\tilde{\Delta}_{f}=\Delta_{0} Z_{f}$. This Kondo-like form of $\mathcal{N}_{f}(\omega)$ holds only for $\omega \ll \tilde{\omega}_{f}$, just like the quasiparticle dispersion makes sense only for $\omega \ll T_{0}$. It cannot be extrapolated to higher frequencies, where the approximation $\Delta(\omega)=i \Delta_{0}$ does not hold. The approximate form given by expression Eq. (43) has a maximum at $\tilde{\omega}_{f}$, where the exact $f$ DOS has a gap; the exact DMFT spectral function has a maximum between $\mu$ and $\tilde{\omega}_{f}$. The width of effective Kondo resonance is $\tilde{\Delta}_{f}$. In the FL regime, $\omega \leq k_{B} T \ll \tilde{\omega}_{f}$, the transport coefficients depend on the characteristic energy scales defined by this effective Kondo resonance.

The width $\tilde{\Delta}_{f}$, like $\tilde{\omega}_{f}$, can be related to the FL scale $T_{0}$ of the periodic Anderson model. Equating the $f$-DOS and the effective single impurity DOS at $\omega=0$ yields

$$
\pi \Delta \mu \mathcal{V} \mathcal{N}_{c}^{0}\left(\mu_{L}\right)=\frac{x}{1+x^{2}}
$$

where $x=\tilde{\omega}_{f} / \tilde{\Delta}_{f}$. Solving for $x$ produces the result

$$
\tilde{\Delta}_{f}=\frac{2 \pi \Delta \mu \mathcal{V} \mathcal{N}_{c}^{0}\left(\mu_{L}\right)}{1 \pm \sqrt{1-\left(2 \pi \Delta \mu \mathcal{V} \mathcal{N}_{c}^{0}\left(\mu_{L}\right)^{2}\right)}} \tilde{\omega}_{f}
$$

and we choose the negative sign. Since $\Delta \mu \mathcal{V} \mathcal{N}_{c}^{0}\left(\mu_{L}\right)$ was shown to be very small [see the discussion around Eqs. (35) and (36)] the root in the above expression can be expanded to produce the lowest order result,

$$
\tilde{\Delta}_{f}=\frac{\tilde{\omega}_{f}}{\pi \Delta \mu \mathcal{V} \mathcal{N}_{c}^{0}\left(\mu_{L}\right)} \simeq \frac{1}{2 \pi} N k_{B} T_{0} .
$$

The above expression, which follows from the DMFT condition, ensures that that the initial slope of the renormalized $f$ DOS is very small,

$$
\left[\frac{\partial \mathcal{N}_{f}(\omega)}{\partial \omega}\right]_{\omega=0} \simeq \frac{\tilde{\omega}_{f}}{\tilde{\Delta}_{f}} \simeq \pi \Delta \mu \mathcal{V} \mathcal{N}_{c}^{0}\left(\mu_{L}\right) \ll 1 .
$$

Finally, we remark that the FS average of the unrenormalized velocity squared, $v_{\mathbf{k}}^{2}$, can be found from the integral

$$
v_{F}^{2}=\left\langle v_{\mathbf{k}_{F}}^{2}\right\rangle=\int d^{d} \mathbf{k} \delta\left(\mathbf{k}-\mathbf{k}_{F}\right) v_{\mathbf{k}}^{2},
$$

where the $\delta$-function restricts the integral to the renormalized FS. In infinite dimensions $v_{F}^{2}$ is a constant for all fillings but in lower dimensions the change of the Fermi volume with pressure or temperature can modify $v_{F}^{2}$ and affect thermal transport. Numerical calculations for the 3-dimensional periodic Anderson model with nearest-neighbor hopping on a simple cubic lattice give $v_{F}^{2}=\left(t a_{l} / \hbar\right)^{2} v^{2}$ where $v^{2} \simeq 1.4$ for $n \simeq 1 / 2$ and $v^{2} \ll 1$ for $n \simeq 1$. 


\section{THE FERMI LIQUID LAWS}

The transport coefficients of the periodic $S U(N)$ Anderson model with infinite correlation between $f$ electrons are obtained in the FL regime by making the lowest order Sommerfeld expansion of transport integrals, Eq. (6), and expressing the integrand $\Lambda(\omega)$ in terms of the known expressions for $v_{k_{F}}^{2}, \mathcal{N}_{c}(\omega)$, and $\tau(\omega, T)$. The algebra is straightforward (for details see Appendix [B]), and yields the transport coefficients as simple powers of reduced temperature $T / T_{0}$.

The specific resistance of $N$ parallel channels obtained in such a way is

$$
\rho(T)=\frac{9 \pi^{3} \mathcal{V}}{\hbar e^{2} v_{F}^{2} N(N-1)\left[N \mathcal{V} \mathcal{N}_{c}^{0}\left(\mu_{L}\right)\right]^{2}}\left(\frac{T}{T_{0}}\right)^{2}
$$

which holds for $N \geq 2$ and arbitrary $\mathcal{N}_{c}^{0}(\omega)$.

The resistivity expression in Eq. (49) neglects terms of the order of $\left(T / T_{0}\right)^{4}$ and is only valid for $T \ll T_{0}$. Even in that temperature range, $\rho(T)$ deviates from the universal (KW) form, because the pre-factor of the $\left(T / T_{0}\right)^{2}$ term has an explicit parameter dependence. The value of these parameters depends o the Fermi volume of the system, which can be changed in several ways. For example, additional impurity scattering or lattice expansion (negative pressure) can localize the $f$ states and exclude them from the Fermi volume. Another possibility is to increase the effective degeneracy of the $f$ states (by pressure or thermal population of the excited states), which changes the number of resonant channels and shifts the Fermi surface away closer to the zone center. The 'jumps' in the Fermi volume changes $\mu_{L}$ and $v_{F}^{2}$, which has a strong impact on the resistivity. This feature can be used to explain the resistivity anomalies that accompany the localization or delocalization of $f$ electrons in heavy fermions, i.e., the breakdown or the formation of the QP bands. The rapid change of the coefficient of the $T^{2}$-term in the resistivity following the pressure- or doping-induced 'jump' of the Fermi volume in Cerium compounds, is discussed in more detail below. On the other hand, if we tune the model parameters of the $S U(N)$ model in such a way that the Fermi volume is preserved (by keeping $N / n$ constant), the Luttinger theorem ensures that $\mu_{L}$ and $v_{F}^{2}$ do not change. In that case, the pre-factor of $\left(T / T_{0}\right)^{2}$ is constant but $\rho(T)$ changes due to variations in $T_{0}$ which can be exponentially fast.

For large $N$, the approximation $N \mathcal{V} \mathcal{N}_{c}^{0}\left(\mu_{L}\right) \simeq n_{f} / \Delta \mu$ yields the expression

$$
\rho(T) \simeq \frac{81\left(\Delta \mu / n_{f}\right)^{2} \mathcal{V}^{3}}{\hbar N(N-1) \pi k_{B}^{2} e^{2} v_{F}^{2}}(\gamma T)^{2}
$$

which simplifies the discussion of heavy fermions in the Kondo limit, $n_{f} \simeq 1$. The Luttinger theorem ensures $n_{f} / \Delta \mu$ is constant even when the tuning of the external parameters gives rise to a charge transfer between the $f$ and $c$ states and thereby changes the renormalized chemical potential. As long as the tuning does not affect the degeneracy of the ground state, the pre-factor of the $(\gamma T)^{2}$-term of the resistivity is always the same.

The Seebeck coefficient is obtained by using the Sommerfeld expansion for $L_{12}$ and $L_{11}$ which gives

$$
\alpha(T) \simeq \frac{4 \pi^{2} k_{B}}{|e| N \Delta \mu \mathcal{V N} \mathcal{N}_{c}^{0}\left(\mu_{L}\right)} \frac{T}{T_{0}} .
$$

The enhancement of $L_{12}$ is solely due to the real part of the self energy. The imaginary part corrects $L_{11}$, and gives a factor $(3 / 2)$ enhancement of $\alpha(T)$ with respect to the $U=0$ case. This factor does not arise in mean field theory or in slave boson approximations based on quadratic Hamiltonians with renormalized parameters 14 . The above result corrects the expression used in our previous paper ${ }^{18}$, which was derived neglecting the energy dependence of the density of states. The term $\Delta \mu$, which does not occur in the low-temperature resistivity expression in Eq. (49), has an explicit parameter dependence, so that, strictly speaking, $\alpha(T)$ is not a universal function of $T / T_{0}$. In bad metals and systems with a lowcarrier concentration, $\mu_{L}$ is close to the band edge, where $\mathcal{N}_{c}^{0}\left(\mu_{L}\right)$ could be very small, so that $\alpha(T)$ could be very large.

The Seebeck coefficient of heavy fermions with $n_{f} \simeq 1$ and large $N$ assumes the simple form

$$
\alpha(T)=\mp \frac{4 \pi^{2} k_{B}}{n_{f}|e|} \frac{T}{T_{0}} .
$$

Since the doubly occupied $f$ states are removed from the Hilbert space, the model is highly asymmetric, and the initial slope $\lim _{T \rightarrow 0} \alpha(T) / T$ never vanishes. As a matter of fact, the closer the system is to half-filling, the larger is the slope, $\alpha / T \propto 1 / T_{0}$. This, however, does not necessarily imply a large thermopower, since the FL laws are only valid for $T \ll T_{0}$ and close to half-filling $T_{0}$ is exponentially small.

The FL result for the thermal conductivity in the clean limit reads

$$
\kappa(T)=T \sigma(T) \mathcal{L}_{0}(T),
$$

where the usual Lorentz number, $\mathcal{L}_{0}=\pi^{2} k_{B}^{2} / 3 e^{2}$, has been replaced by the effective one,

$$
\mathcal{L}_{0}(T)=\overline{\mathcal{L}}_{0}\left[1-\frac{32 \pi^{2}}{n_{f}^{2}}\left(\frac{T}{T_{0}}\right)^{2}\right],
$$

and $\overline{\mathcal{L}}_{0}=\pi^{2} k_{B}^{2} / 2 e^{2}$. This change is due to the imaginary part of the self energy and therefore not obtainable by mean-field or slave-boson calculations which neglect the QP damping. The $T \rightarrow 0$ limit yields the WiedemannFranz law, $\kappa(T) \propto T \sigma(T)$, but the correction given by the square bracket leads to deviations even at low temperatures. Since the factor multiplying the $T^{2}$ term is very large, we find a reduction of $\kappa(T)$ and substantial deviations from the WF law much below $T_{0}$. 
Before closing this section, we summarize the procedure for calculating the transport coefficients of the periodic Anderson model in the FL regime. The model is specified by the hybridization $V$, the bare $f$-level position $E_{f}$, the degeneracy $N$, the center of the bare conduction band $E_{0}$, the unperturbed dispersion $\epsilon_{\mathbf{k}}$, and the restriction $n_{f} \leq 1$. The unperturbed density of conduction states $N_{c}^{0}(\omega)$ is easily obtained from $\epsilon_{\mathbf{k}}$. For a given total electron density $n$ (per cell) we use the DMFT+NRG or some simpler scheme, like the slave bosons, to find the renormalized chemical potential, the number of $f$ electrons, and the low-temperature coefficient of the specific heat which sets the FL scale $T_{0}$. These static quantities can be calculated in thermal equilibrium to a very high accuracy. For constant hybridization, we can make separate DMFT+NRG runs for $n_{c}(\mu)$ and $n_{f}(\mu)$ and do not have to calculate the spectral function which is not given very accurately (for all $\omega$ ) by the NRG. From $n=N n_{c}+n_{f}$, we obtain $\mu, \mu_{L}$, and $\Delta \mu$, which provide the renormalized FS and the average $v_{F}^{2}$. From $T_{0}, \mu_{L}$, $\Delta \mu$ we obtain the Kondo scale $\tilde{\omega}_{f}$ and specify completely the low-energy behavior of $N_{c}(\omega)$ and $\tau(\omega)$. The FL laws follow at once. For heavy fermions with large $N, n_{f} \simeq 1$ and small $\tilde{V}$, the procedure simplifies considerably, since we can approximate $\Delta \mu N \mathcal{V} \mathcal{N}_{c}^{0}\left(\mu_{L}\right) \simeq n_{f}$, and obtain all the renormalized quantities using $\tilde{\omega}_{f} \simeq n_{f} k_{B} T_{0} / 2$.

\section{DISCUSSION OF EXPERIMENTAL RESULTS}

\section{A. The universal ratios}

The FL laws in Eqs. (49 53) describe coherent charge and heat transport in stoichiometric compounds, in a way analogous to the phase-shift expressions for dilute Kondo alloys 19 . They explain the near-universal behavior of the KW ratio ${ }^{8} 10$ reported for many heavy xfermions and valence fluctuators. The ratio $\rho(T) /(\gamma T)^{2}$ obtained from Eq. (49) exhibits an explicit dependence on the ground state degeneracy and the average velocity (squared), and an implicit dependence on the Fermi volume, i.e., on the carrier concentration. The $N$-dependence as well as the dependence on carrier concentration of the $\mathrm{KW}$ ratio were recently emphasized by Kontani and his collaborators ${ }^{9.10}$, who obtained the power law $n^{-4 / 3}$ for the latter by using the free electron approximation for the average velocity and the density of states in the expression for the resistivity. Our formulation leads to a similar $\mathrm{N}$ dependence [see also Eq. (50)] but includes also the dependence on the average velocity, the renormalized Fermi volume and the carrier concentration, which are implicit functions of the degeneracy and have to be taken into account when discussing the pressure or doping experiments on strongly correlated electron systems 11 .

As regards the thermal transport, Eq. (51) gives $q=$ $\lim _{T \rightarrow 0}|e \alpha| / \gamma T \simeq 12 /\left[N \Delta \mu \mathcal{N}_{c}^{0}\left(\mu_{L}\right)\right]$, which has an explicit parameter dependence, so that the $q$-ratio, like the
KW ratio, can deviate from the universal value $\frac{1,2}{2}$. In bad metals and systems with a low-carrier concentration, $\mu_{L}$ is close to the band edge, where $\mathcal{N}_{c}^{0}\left(\mu_{L}\right)$ could be very small, making $\alpha(T)$ large. For a given system, the $q$-ratio can be pressure dependent due to the transfer of $f$ electrons into conduction band. The data on the pressure dependence are not available but the deviations from the universal value are indicated by recent chemical pressure data 2 .

A further remarkable consequence of correlations is the enhancement of the low-temperature figure-of-merit due to the deviations from the WF law. Using Eq. (53) and neglecting phonons in the FL regime we express the figure-of-merit as a ratio $Z T=\alpha^{2}(T) / \mathcal{L}(T)$. For constant Lorenz number, the maximum of $Z T$ is defined by the thermopower but in correlated systems the temperature dependence of the effective Lorenz number can lead to an additional enhancement. Even though our FL result is valid only for $T \ll T_{0}$, it captures the essential features: an increase of $\alpha^{2}$ and a decrease of $\mathcal{L}(T)$ that ultimately give rise to $Z T>1$. The enhancement of $Z T$ is due to the renormalization of the thermopower and the Lorentz number i.e., $Z T>1$ is not restricted to metallic systems with $\alpha(T)>155 \mu \mathrm{V} / \mathrm{K}$. We expect large $Z T$ for small $T_{0}$ but to find the optimal situation one should tune the parameters and study the border of the FL regime by numerical methods.

\section{B. Pressure dependence of the low-temperature resistivity}

The FL laws derived for the periodic Anderson model explain the changes observed in the transport coefficients of heavy fermions under applied pressure. As an illustration, we consider the pressure dependence of the coefficient of the $T^{2}$ term in the resistivity, defined as $A(p)=\left(\rho-\rho_{0}\right) / T^{2}$, where $\rho_{0}$ is the residual resistivity. In the case of the two heavy fermion antiferromagnets $\mathrm{CePd}_{2-x} \mathrm{Ge}_{2-x}{ }^{23}$ and $\mathrm{CeRu}_{2} \mathrm{Ge}_{2}{ }^{24}, A(p)$ is small and nearly pressure independent for $p \leq 4 \mathrm{GPa}$. Above $4 \mathrm{GPa}, A(p)$ increases rapidly and reaches a maximum value for pressure between 4 and 8 and GPa. At the critical pressure, $p_{c}$, the maximum of $A\left(p_{c}\right)$ is typically one order of magnitude higher than at ambient pressure. For $p>p_{c}$, the value of $A(p)$ drops to the ambient pressure one. A somewhat different behavior is found in the heavy fermion superconductor $\mathrm{CeCu}_{2} \mathrm{Si}_{2}$ and in the antiferromagnet $\mathrm{CeCu}_{2} \mathrm{Ge}_{2}$, when analyzed in the normal state $\frac{21,22}{2}$. The values of $A(p)$ are large at initial pressure which is applied in order to restore the FL behavior; above that pressure $A(p)$ decreases to a plateau and then drops by nearly two orders of magnitude ${ }^{21,22}$.

A sharp maximum of $A(p)$ is observed for systems in which the ground state of the $4 f$ ion is characterized at ambient pressure by a $\mathrm{CF}$ doublet well separated from the excited CF states. The Neel temperature of such systems is much higher than the Kondo temperature and 
large paramagnetic entropy of Ce ions is removed at $T_{N}$ by an AFM transition rather than by a Kondo crossover. The low-entropy state involves large unscreened moments which correspond to the $4 f$ ions frozen in the hightemperature (magnetic) configuration. Below $T_{N}$, the conduction electrons are essentially free, except for some magnon and impurity scattering, such that the resistivity at ambient pressure is small and weakly temperaturedependent, as shown by $\mathrm{CePd}_{2-x} \mathrm{Ge}_{2-x}$ and $\mathrm{CeRu}_{2} \mathrm{Ge}_{2}$ data.

To explain the experimental data we assume that an increase of pressure leads at $p_{c}$ to the delocalization of the $4 f$ states $^{28}$ and the formation of hybridized bands, which can be described by the $S U(2)$ Anderson model. The two channels (sub-bands) in which the lowest $4 f$ doublet hybridizes with the conduction states of the appropriate symmetry, accommodate nearly one electron per site, $n_{c}+n_{f} / 2 \simeq 1$, for each flavor. The renormalized Fermi volume is large and the FS is close to the edge of the Brillouin zone, where $v_{F}^{2}$ is small. The corresponding value of $\mu_{L}$ is of the order of the bandwidth [see Eqs. (31) and (32)], such that $\mathcal{N}_{c}^{0}\left(\mu_{L}\right)$ is also small. [The maximum of $\mathcal{N}_{c}^{0}(\omega)$ is assumed to be close to the center of the band.] Since $T_{0}$ is small $\left(T_{0}<T_{N}\right)$, the coefficient of the $T^{2}$ term in the resistivity, given by Eq. (49) for $N=2$, is large. The enhancement of $A(p)$ with respect to the values at ambient pressure (where the $f$ states are localized) is due to the delocalization of the $f$ states by applied pressure. In a system with delocalized $f$ states, the main effect of pressure is to increase the hybridization and $T_{0}$, provided the degeneracy of the lowest occupied CF level is preserved. We can understand the increase of $T_{0}$ by recalling that the FL scale of the lattice is proportional to the low-energy scale of the auxiliary impurity model [see Eq. (34)] and that an increase of the Kondo scale with hybrdization (pressure) is a typical feature of any Kondo system. An increases of $T_{0}$ can also be inferred from the experimental data ${ }^{21,22}$, which show that in $\mathrm{CePd}_{2-x} \mathrm{Ge}_{2-x}$ and $\mathrm{CeRu}_{2} \mathrm{Ge}_{2}$ the value of $T_{0}$ scales with the high-temperature Kondo scale.

A qualitative change occurs at the point where the hybridization becomes so large that the system cannot sustain the CF excitations and the degeneracy of the $f$ level changes from two to six. To estimate the resistivity of this high-pressure state we use the $S U(6)$ model in which a single $f$ electron is distributed over six equivalent hybridized channels. In that case, our FL solution shows that the FS is shifted away from the zone boundary, $\mu_{L}$ is decreased and $\mathcal{N}_{c}^{0}\left(\mu_{L}\right)$ increased, with respect to the values obtained for $N=2$. The average squared velocity $v_{F}^{2}$ and the FL scale $T_{0}$ are also increased for $N=6$, which reduces $A(p)$ to small values, in agreement with the experimental data $21,22,23,24$. The drop of $A(p)$ for $p>p_{c}$ signifies the doublet-sextet crossover and is mainly due to the pre-factor in Eq. (49), which has an explicit and implicit dependence on $N$. At the crossover, the Fermi volume changes and the KW ratio is strongly pressure dependent. Once the degeneracy of the $f$ state is stabilized at a higher value, a further increase of pressure reduces $A(p)$ by increasing $T_{0}$ but does not change $v_{F}^{2}$ or $\mu_{L}$, which are fixed by the Luttinger theorem for $\left(n_{c}+n_{f} / 6\right)$ states per channel. Eventually, at very high pressure, the system is transformed into a valence fluctuator with an enormously enhanced FL scale, such that $A(p)$ drops to a very small value.

As regards $\mathrm{CeCu}_{2} \mathrm{Si}_{2}{ }^{22}$ and $\mathrm{CeCu}_{2} \mathrm{Ge}_{2}{ }^{21}$, we can explain the data using the same reasoning as above, if we assume that the $f$ states are delocalized at the initial pressure which restores the FL state. The value of $A(p)$ at the initial pressure is large, because the temperature dependence of the resistivity is due to two degenerate sub-bands with very heavy fermions. The FS of these sub-bands is close to the zone boundary, where $v_{F}^{2}, \mu_{L}$, and $\mathcal{N}_{c}^{0}\left(\mu_{L}\right)$ are very small. The hybridized bands which involve the excited CF states have a small Fermi volume and their low-energy properties are free-electron like. They carry most of the current but have temperatureindependent resistivity and do not affect $A(p)$. The first decrease of $A(p)$ occurs at pressure at which an increase of the hybridization removes the CF excitations, such that the effective degeneracy of the $f$ state increases from 2 to 4 or 6 . For large $N$, the expression in Eq. (49) gives small $A$. The final drop of $A(p)$ is due to the crossover into the valence fluctuating regime and an exponential increase of the FL scale $T_{0}$. If we assume a proportionality between $T_{0}$ and the Kondo scale $T_{K}$, which is indicated by the experimental data, our calculations would explain the $A(p)$ versus $T_{K}$ scaling reported in Ref. 22 .

The above scenario also offers an explanation for the peak observed in the residual resistivity of $\mathrm{CeCu}_{2} \mathrm{Ge}_{2}{ }^{21}$ and $\mathrm{CeCu}_{2} \mathrm{Si}_{2}{ }^{22}$ for pressures at which the degeneracy of the $f$ level changes and $A(p)$ drops from the maximum value. At such a pressure, the formerly non-resonant channels responsible for the low residual resistivity transform into resonant ones, which reduces $v_{F}^{2}$ and $\mathcal{N}_{c}^{0}\left(\mu_{L}\right)$. For a constant impurity scattering rate, this leads to a drastic increase of $\rho_{0}$. A further increase of pressure transforms the system into a valence fluctuator with nearly free conduction states and small $\rho_{0}$. An alternative explanation for this peak has been suggested by Miyake and Maebashi ${ }^{29}$, who attribute it to critical valence fluctuations. In our approach, these would occur at higher pressure, and we expect the resulting feature to be narrower than the one observed experimentally. A peak in $\rho_{0}(p)$ is also observed in $\mathrm{YbCu}_{2} \mathrm{Si}_{2}{ }^{21}$. In this case, the mechanism that leads to it is the "mirror-image" of the preceding one ${ }^{30}$ : starting from a valence fluctuating regime at ambient pressure, the system is driven into a state with a well defined valence in which all eight components of the $J=7 / 24 f$-multiplet hybridize with conduction states, so that $\rho_{0}$ is very large. A further increase of pressure stabilizes the magnetic $4 f^{13}$ configuration $^{31}$, inhibits the charge fluctuations, and gives rise to the $\mathrm{CF}$ excitations. This reduces the effective degeneracy of the $f$ hole by splitting-off the doubly-degenerate 'resonant' sub-band with heavy QPs from the "non-resonant" sub- 
bands with nearly free electrons. For the reasons given above, $\rho_{0}$ is reduced yet larger than at ambient pressure.

\section{SUMMARY AND CONCLUSIONS}

A detailed understanding of the thermoelectric properties of the periodic Anderson model in the full temperature range and for arbitrary parameters is of a considerable interest, as it might facilitate the search for new thermoelectrics with a useful low-temperature figure-ofmerit. The model with the $S U(N)$ symmetry and an infinitely large Coulomb repulsion between $f$ electrons captures the low-temperature features of the intermetallic compounds with $\mathrm{Ce}, \mathrm{Eu}$ and $\mathrm{Yb}$ ions which exhibit often a large thermopower and might have a potential for applications.

The thermoelectric response of the $S U(N)$ model with $n=N n_{f}+n_{c}$ particles is calculated in the FL regime assuming that the FL scale $T_{0}$ is known. The value of $T_{0}$ can be obtained from the numerical DMFT results for the linear coefficient of the specific heat or estimated from an analytic expression for the QP DOS given by the slave-boson approximation ${ }^{32}$. The transport integrals are related to the current-current correlation function and calculated by using the Luttinger theorem and the DMFT condition. The low-temperature transport coefficients are obtained from the Sommerfeld expansion as power series in terms of the reduced temperature $T / T_{0}$. The coefficients in the expansion depend on the average conduction electron velocity, the unit cell volume, the effective degeneracy of the $f$ state, the unrenormalized density of $c$ states, $\mathcal{N}_{c}^{0}\left(\mu_{L}\right)$, and the shift in the chemical potential $\Delta \mu=\mu_{L}-\mu$ which also has a simple physical interpretation. The chemical potential $\mu$ corresponds to $n$ interacting particles and for a flat low-energy dispersion, $\mu$ is not much different from the chemical potential of $N n_{c}$ conduction states decoupled from the $f$ states. The FS of such a conduction band encloses $N n_{c}$ points in the $\mathbf{k}$-space and is considered to be 'small'. The FS of the Anderson model, determined by the Luttinger theorem, accommodates $f$ electrons in addition to $c$ electrons and is considered to be 'large'. For k-independent hybridization, this 'large' FS coincides with the FS of a free conduction band with $n$ electrons and chemical potential $\mu_{L}$. Obviously, the FS of a non-interacting conduction band with $n$ electrons differs from the FS of $N n_{c}$ electrons, so that the shift $\Delta \mu$ measures the 'jump' in the Fermi volume due to the hybridization.

The parameter dependence of the coefficients multiplying various powers of reduced temperature corrects the simple scaling behavior and explains the deviations of the KW and the $q$-ratio from the universal constants. The FL law for the conductivity gives the $\mathrm{KW}$ ratio which depends on the multiplicity of the $f$ state, the unit cell volume, the average FS velocity, and the carrier concentration, in agreement with the experimental data 11 . The FL law for the thermopower gives the $q$-ratio which de- pends on the concentration of $f$ electrons. From these results we conclude that, in some systems, pressure or chemical pressure can cause a substantial shift of the 'universal ratios' from the common values. We also find that the quasiparticle damping leads to the break-down of the WF law due to the temperature dependence of the effective Lorenz number. In the absence of the thermal current due to phonons, this would lead to a substantial enhancement of the thermoelectric figure-of-merit $Z T>1$. Assuming that pressure changes the hybridization and the effective degeneracy of the $f$ state we explained the pronounced maximum of the coefficient of the $T^{2}$ term of the electrical resistance $21,22,23,24$ and the rapid variation of residual resistance ${ }^{21,22}$ found in a number of $\mathrm{Ce}$ and $\mathrm{Yb}$ intermetallics at some critical pressure.

Our results describe the main features of thermal transport that one finds in heavy fermions in the FL regime but do not indicate the temperature at which the FL description breaks down nor relate the FL scale to the Kondo scale, which characterizes the high-temperature behavior. A complete discussion should explain the lowand high-temperature behavior on equal footing, provide all the relevant energy scales of the system, and account for the change in the effective degeneracy of the lowenergy states due to applied pressure, doping or temperature. The change of the effective degeneracy can have a dramatic effect on the transport coefficients and seems to be responsible for complicated thermoelectric response of intermetallic compounds with $\mathrm{Ce}$ and $\mathrm{Yb}$ ions. The realistic modeling should remove the $S U(N)$ symmetry, take into account the excited $\mathrm{CF}$ states, consider the details of the band structure, and/or include aditional interactions. Unfortunately, none of the presently available methods can solve such more realistic models, explain the crossovers between various physical states, and describe the behavior of the correlation functions in the full temperature and pressure range.

A rough description of the high-temperature regime, which takes into account the CF splitting, can be obtained by assuming that the conduction electrons scatter incoherently on the $4 f$ ions. This reduces the lattice model with $n$ electrons per unit cell to an effective Anderson impurity model which can be solved very accurately by the NCA 18,30 . The NCA solution leads to an exponentially small Kondo scale and explains the main features that one finds in the thermoelectric response of heavy fermions and valence fluctuators for $T \geq T_{K}$. In particular, the NCA shows that the effective degeneracy of the model changes as the excited CF become thermally populated. Some justification for applying an effective single impurity model to the stoichiometric compounds is provided by the fact that in the temperature range we are concerned with the resistivity of most heavy fermions is very large and the mean free path is not much longer than a few lattice spacings. Furthermore, the NCA solution for an effective two-fold degenerate impurity model ${ }^{18,30}$ agrees for $T \geq T_{K}$ with recent $\mathrm{DMFT}+\mathrm{NRG}$ results for the two-fold degenerate periodic Anderson mode ${ }^{16}$. For 
an $N$-fold degenerate model the NCA gives $\alpha(T)$ and the power factor $P(T)=\alpha^{2}(T) / \rho(T)$ with a pronounced maximum and $\mathcal{L}(T)$ with a shallow minimum around $T_{K}$, which supports our previous conclusions regarding the enhancement of the thermoelectric figure-of-merit. The agreement between the perturbative solution of an effective single impurity model and the experimental data indicates that the high-temperature state of heavy fermions and valence fluctuators can be represented by a nearly free conduction band which is weakly perturbed by localized (paramagnetic) $f$ states. Ignoring the thermal broadening of the Fermi distribution function we find that this FS is much smaller than the low-temperature one which must include the $f$ electrons in order to satisfy the Luttinger theorem. The perturbative solution breaks down in the coherent regime, as it cannot describe the change in the Fermi volume. For a periodic model, the reduction of the paramagnetic entropy, the crossover from the high-temperature perturbative regime into the FL regime, and relationship between $T_{K}$ and $T_{0}$ can only be obtained by non-perturbative methods.

The DMFT+NRG method describes, in principle, the crossover from the high-entropy state formed above $T_{K}$ to the low-entropy FL ground state state. It provides the Kondo scale $T_{K}$ which governs the high-temperature behavior and gives an accurate numerical estimate of the FL scale $T_{0}$ and other thermodynamic quantities, like the number of particles or the chemical potential of the ground state. However, neither the DMFT+NRG nor the effective impurity approach provide a quantitative description of the transport properties of the periodic Anderson model for temperatures below $T_{K}$ or $T_{0}$. The usefulness of the FL approach is that it gives the lowtemperature transport coefficients for a given $T_{0}$ and allows us to obtain an overall description by extrapolating between the FL solution and the high-temperature one obtained by the NCA or the DMFT+NRG methods.

It would be interesting to perform an experimental study of the pressure-induced deviations of the $q$-ratio and the KW ratio from their universal values, using the universal behavior of the power factor or the effective Lorenz number as consistency checks. Since $P(T)$ and $\mathcal{L}(T)$ require only transport measurements, they are well suited for pressure experiments. The above discussion makes clear that pressure experiments provide the most stringent test of the FL laws.

\section{Acknowledgments}

We thank David Logan for pointing out some unclear points in the original manuscript. Useful discussion with K. Yamada, D. Jaccard and T. Pruschke are gratefully acknowledged. This work has been supported by the Ministry of Science of Croatia (Grant No. 035-03528432849 ), the COST P-16 ECOM project, and the National Science Foundation under Grant Nos. DMR-0210717 and DMR-0705266. The work at SISSA has been supported by the Central European Initiative. V.Z. and J.K.F acknowledge the hospitality of the ETH Zurich, where this work has been completed.

\section{APPENDIX A: CORRELATION FUNCTION IN THE FERMI LIQUID REGIME}

To find $\Lambda(\omega, T)$ we use the Kubo formula for the static conductivity which provides $L_{11}$ as the zero-frequency limit of the number current -number current correlation function 25

$$
L_{11}^{\alpha \beta}=\lim _{\nu \rightarrow 0} \operatorname{Re} \frac{i}{\nu} \bar{L}_{11}^{\alpha \beta}(\nu)
$$

where

$$
\bar{L}_{11}^{\alpha \beta}\left(i \nu_{l}\right)=\frac{\hbar}{\mathcal{V} \mathcal{N}_{i}} \int_{0}^{\beta} d \tau e^{i \nu_{l} \tau}\left\langle T_{\tau} \mathbf{j}_{\alpha}^{\dagger}(\tau) \mathbf{j}_{\beta}(0)\right\rangle
$$

$\nu_{l}=2 \pi k_{B} T l$ is the Bosonic Matsubara frequency, the $\tau$ dependence of the operator is with respect to the Hamiltonian in Eq. (11), the subscripts $\alpha$ and $\beta$ denote the respective spatial indices of the number-current vector $\mathbf{j}_{c} / e$, and we must analytically continue $\bar{L}_{11}^{\alpha \beta}\left(i \nu_{l}\right)$ to the real axis $\bar{L}_{11}^{\alpha \beta}(\nu)$ before taking the limit $\nu \rightarrow 0$.

Substituting the definition of the particle current operator from Eq. (5) into Eq. (A2) for $\bar{L}_{11}^{\alpha \beta}$ and taking the limit of infinite dimensions, in which the dressed correlation function is equal to the bare one ${ }^{33,34}$, we obtain

$\bar{L}_{11}^{\alpha \beta}\left(i \nu_{l}\right)=\frac{-\hbar}{\mathcal{V} \mathcal{N}_{i}} \int_{0}^{\beta} d \tau e^{i \nu_{l} \tau} \sum_{\mathbf{k}} \mathbf{v}_{\mathbf{k} \alpha} \mathbf{v}_{\mathbf{k} \beta} G_{c}(\mathbf{k}, \tau) G_{c}(\mathbf{k},-\tau)$.

where $G_{c}(\mathbf{k}, \tau)$ is the imaginary time Green's function of the $c$ electrons, which can be expressed as a Fourier series $G_{c}(\mathbf{k}, \tau)=k_{B} T \sum_{n} \exp \left(-i \omega_{n} \tau\right) G_{c}\left(\mathbf{k}, i \omega_{n}\right)$. Substituting into Eq. (A3) and integrating over imaginary time provides the result

$$
\begin{array}{r}
\bar{L}_{11}^{\alpha \beta}\left(i \nu_{l}\right)=\frac{-\hbar}{\mathcal{V} \mathcal{N}_{i}} k_{B} T \sum_{n} \sum_{\mathbf{k}} \mathbf{v}_{\mathbf{k} \alpha} \mathbf{v}_{\mathbf{k} \beta} \\
G_{c}\left(\mathbf{k}, i \omega_{n}\right) G_{c}\left(\mathbf{k}, i \omega_{n}+i \nu_{l}\right)
\end{array}
$$

which has to be analytically continued to the real axis. Since the Green's functions in Eq. (A4) depend on $\mathbf{k}$ only through $\epsilon(\mathbf{k})$ which is an even function of $\mathbf{k}$, while $\mathbf{v}_{\mathbf{k} \alpha}$ is odd, the summation over $\mathbf{k}$ vanishes for $\alpha \neq \beta$. We consider only isotropic systems, where $L_{11}^{\alpha \alpha}=L_{11}$. To perform the analytic continuation we follow closely Ref. 35 and obtain the result (evaluated explicitly for a threedimensional system)

$$
\begin{gathered}
L_{11}=\frac{-\hbar}{\mathcal{V} \mathcal{N}_{i}} \frac{v_{F}^{2}}{3 \pi} \int_{-\infty}^{\infty} d \omega \lim _{\nu \rightarrow 0} \frac{f(\omega)-f(\omega+\nu)}{\nu} \\
\times \operatorname{Re}\left[\frac{G_{c}(\omega)-G_{c}(\omega+\nu)}{\nu+\Sigma_{c}(\omega)-\Sigma_{c}(\omega+\nu)}-\frac{G_{c}^{*}(\omega)-G_{c}(\omega+\nu)}{\nu+\Sigma_{c}^{*}(\omega)-\Sigma_{c}(\omega+\nu)}\right] .
\end{gathered}
$$


where we introduced the local Green's function

$$
G_{c}(\omega)=\sum_{\mathbf{k}} G_{c}(\mathbf{k}, \omega),
$$

and replaced the square of the $\alpha$-component of velocity by its Fermi surface average $v_{F}^{2} / d$ ( $d$ is the spatial dimension, which we can take to be equal to 3 for real systems). This step is justified, because the energy integrations in Eq. (A5) is restricted to a narrow interval around the Fermi energy, where the integrand is singular, so that the main contribution to the $\mathbf{k}$ summation comes from the $\mathbf{k}$-points close to the Fermi surface; on the infinitedimensional hypercubic lattice, the integral can be performed exactly and one finds that the average square velocity is equal to $a_{l}^{2} t^{* 2} / 16 \hbar^{2}$ when expressed in terms of the reduced nearest-neighbor hopping $t^{*}=2 \sqrt{d} t$.

We can now take the limit of $\nu \rightarrow 0$. Writing

$$
\lim _{\nu \rightarrow 0} \frac{G_{c}(\omega)-G_{c}(\omega+\nu)}{\nu+\Sigma_{c}(\omega)-\Sigma_{c}(\omega+\nu)}=-\frac{\partial G_{c}(\omega)}{\partial \omega} \frac{1}{1-\frac{\partial \Sigma_{c}(\omega)}{\partial \omega}}
$$

and

$$
\lim _{\nu \rightarrow 0} \frac{G_{c}^{*}(\omega)-G_{c}(\omega+\nu)}{\nu+\Sigma_{c}^{*}(\omega)-\Sigma_{c}(\omega+\nu)}=\frac{\operatorname{Im} G_{c}(\omega)}{\operatorname{Im} \Sigma_{c}(\omega)}
$$

produces our final result

$$
L_{11}=\int_{-\infty}^{\infty} d \omega\left(-\frac{d f(\omega)}{d \omega}\right) \Lambda(\omega, T)
$$

where $\Lambda(\omega, T)$ is defined by

$$
\Lambda(\omega)=\frac{\hbar}{\mathcal{V} \mathcal{N}_{c}} \frac{v_{F}^{2}}{3 \pi}\left(\frac{\operatorname{Im} G_{c}(\omega)}{\operatorname{Im} \Sigma_{c}(\omega)}+\operatorname{Re}\left[\frac{\frac{\partial G_{c}(\omega)}{\partial \omega}}{1-\frac{\partial \Sigma_{c}(\omega)}{\partial \omega}}\right]\right) .
$$

To estimate the relative importance of the two terms in Eq. A10 we introduce the Hilbert transform of $\mathcal{N}_{c}(\omega)$,

$$
\mathcal{H}_{c}(\omega)=-\frac{1}{\pi \mathcal{V} \mathcal{N}_{i}} \operatorname{Re} G_{c}(\omega)
$$

In the FL regime, where $\mathcal{N}_{c}(\omega)$ is $\delta$-function like, the slope of $\mathcal{H}_{c}(\omega)$ is very large; it is proportional to the $c$ electron enhancement factor

$$
\operatorname{Re}\left[1-\frac{\partial \Sigma_{c}(\omega)}{\partial \omega}\right]=Z_{c}^{-1} .
$$

On the other hand, $\operatorname{Im} \partial \Sigma_{c}(\omega) / \partial \omega$ is small around $\omega=0$, because $\operatorname{Im} \Sigma_{c}(\omega)$ is close to its maximum value. Using

$$
\operatorname{Im} \frac{\partial \Sigma_{c}(\omega)}{\partial \omega} \ll Z_{c}^{-1} .
$$

we neglect $\left[\operatorname{Im} \partial \Sigma_{c}(\omega) / \partial \omega\right]^{2}$ in the denominator of the second term for $\Lambda(\omega)$ in Eq. A10 and approximate

$$
\operatorname{Re}\left[\frac{\frac{\partial G_{c}(\omega)}{\partial \omega}}{1-\frac{\partial \Sigma_{c}(\omega)}{\partial \omega}}\right] \simeq Z_{c} \frac{\partial \mathcal{H}_{c}(\omega)}{\partial \omega}-\frac{\partial \mathcal{N}_{c}(\omega)}{\partial \omega} \operatorname{Im} \frac{\partial \Sigma_{c}(\omega)}{\partial \omega}
$$

This term is small with respect to $\mathcal{N}_{c}(\omega) / \operatorname{Im} \Sigma(\omega)$ which diverges in the limit $T, \omega \rightarrow 0$. Keeping only the singular term in Eq. (A10) we obtain the result of Eq. (7) used in the text.

\section{APPENDIX B: TRANSPORT COEFFICIENTS}

The transport coefficients are obtained by expanding the derivative of the Fermi function in a Sommerfeld expansion, $-\partial f(\omega) / \partial \omega=\delta(\omega)+\left(\pi^{2} k_{B}^{2} T^{2} / 6\right)\left[\partial^{2} \delta(\omega) / \partial \omega^{2}\right]$, which gives the following result for the transport integrals

$$
\begin{aligned}
L_{m n} & =\left[\omega^{m+n-2} \Lambda(\omega, T)\right]_{\omega=0} \\
& +\frac{\pi^{2} k_{B}^{2} T^{2}}{6}\left\{\frac{\partial^{2}}{\partial \omega^{2}}\left[\omega^{m+n-2} \Lambda(\omega, T)\right]\right\}_{\omega=0} .
\end{aligned}
$$

For thermal transport we have to evaluate

$$
\begin{aligned}
L_{11} & =[\Lambda(\omega, T)]_{\omega=0}+\left.\frac{\pi^{2} k_{B}^{2} T^{2}}{6} \Lambda^{\prime \prime}(\omega, T)\right|_{\omega=0}, \\
L_{12} & =\left.\frac{\pi^{2} k_{B}^{2} T^{2}}{3} \Lambda^{\prime}(\omega, T)\right|_{\omega=0}, \\
L_{22} & =\left.\frac{\pi^{2} k_{B}^{2} T^{2}}{3} \Lambda(\omega, T)\right|_{\omega=0},
\end{aligned}
$$

where

$$
\begin{aligned}
\Lambda(\omega, T) & =\frac{1}{3} v_{F}^{2} \mathcal{N}_{c}(\omega) \tau(\omega, T) \\
\Lambda^{\prime}(\omega, T) & =\frac{1}{3} v_{F}^{2}\left[\mathcal{N}_{c}^{\prime}(\omega) \tau(\omega, T)+\mathcal{N}_{c}(\omega) \tau^{\prime}(\omega, T)\right] \\
\Lambda^{\prime \prime}(\omega, T) & =\frac{1}{3} v_{F}^{2}\left[\mathcal{N}_{c}^{\prime \prime}(\omega) \tau(\omega, T)\right. \\
& \left.+2 \mathcal{N}_{c}^{\prime}(\omega) \tau^{\prime}(\omega, T)+\mathcal{N}_{c}(\omega) \tau^{\prime \prime}(\omega, T)\right]
\end{aligned}
$$

and $\Lambda^{\prime}$ and $\Lambda^{\prime \prime}$ denote the first and second derivatives with respect to $\omega$. The first derivative of $\mathcal{N}_{c}(\omega)$ is

$$
\mathcal{N}_{c}^{\prime}(\omega)=\frac{2\left(\omega-\tilde{\omega}_{f}\right)}{Z_{f} \tilde{V}^{2}} \mathcal{N}_{f}(\omega)+\frac{\left(\omega-\tilde{\omega}_{f}\right)^{2}}{Z_{f} \tilde{V}^{2}} \mathcal{N}_{f}^{\prime}(\omega),
$$

and becomes in the $\omega \rightarrow 0$ limit

$$
\mathcal{N}_{c}^{\prime}(0) \simeq-2 \frac{\mathcal{N}_{c}(0)}{\tilde{\omega}_{f}},
$$

where the DMFT condition showed that the derivative of the $f$-electron DOS is small at $\omega=0$. For the second derivative, we obtain

$$
\mathcal{N}_{c}^{\prime \prime}(0)=\frac{2 \mathcal{N}_{c}(0)}{\tilde{\omega}_{f}^{2}},
$$

using $\mathcal{N}_{f}(0) / Z_{f} \tilde{V}^{2}=\mathcal{N}_{c}(0) / \tilde{\omega}_{f}^{2}$ and dropping the terms proportional to $\tilde{\omega}_{f} \mathcal{N}_{f}^{\prime}(0)$ and $\tilde{\omega}_{f}^{2} \mathcal{N}_{f}^{\prime \prime}(0)$ which are exponentially small [see also Eq. (47)].

The transport relaxation time given by the expression in Eq. (39) yields at $\omega=0$

$$
\begin{array}{r}
\tau^{\prime}(0, T)=-2 \frac{\tau_{0}(T)}{\tilde{\omega}_{f}}, \\
\tau^{\prime \prime}(0, T)=2 \tau_{0}(T)\left[\frac{1}{\tilde{\omega}_{f}^{2}}-\frac{1}{\left(\pi k_{B} T\right)^{2}}\right] .
\end{array}
$$


The transport integrals are now easy to find. The first one is obtained from Eq. (B7), which gives

$$
\begin{aligned}
& L_{11} \simeq \frac{1}{3} v_{F}^{2} \mathcal{N}_{c}(0) \tau_{0}(T)\{\{1+ \\
+ & \left.\frac{\pi^{2} k_{B}^{2} T^{2}}{3}\left[\frac{6 \mathcal{N}_{c}(0)}{\tilde{\omega}_{f}^{2}}-\frac{1}{\left(\pi k_{B} T\right)^{2}}\right]\right\} . \\
\simeq & \Lambda(0, T)\left[\frac{2}{3}+\mathcal{O}\left(T^{2}\right)\right] .
\end{aligned}
$$

The last term in the square bracket in the second line grows as $1 / T^{2}$, while the first one is a (large) constant which we neglect at low enough temperature. Since $\Lambda(0, T) \propto 1 / T^{2}$, this approximation amounts to keeping the $T^{2}$ terms in the electrical resistance and neglecting the $T^{4}$ contribution. The correcting factor $2 / 3$ in Eq. (B13) originates from the imaginary part of the self energy and it is well known from the dilute alloy problem ${ }^{36}$; hence it arises only in the clean limit. Inserting the expressions for the renormalized c-DOS [Eq.(27)] and the relaxation time [Eq.(40)], we obtain the dominant lowtemperature contribution to $L_{11}$ :

$$
L_{11}=v_{F}^{2} \frac{\hbar(N-1)\left[N \mathcal{V} \mathcal{N}_{c}^{0}\left(\mu_{L}\right)\right]^{2}}{9 \pi^{3} \mathcal{V}}\left(\frac{T_{0}}{T}\right)^{2}
$$

which yields for the static conductivity $\sigma(T)=N e^{2} L_{11}$

$$
\sigma(T)=\frac{\hbar e^{2} v_{F}^{2} N(N-1)\left[N \mathcal{V} \mathcal{N}_{c}^{0}\left(\mu_{L}\right)\right]^{2}}{9 \pi^{3} \mathcal{V}}\left(\frac{T_{0}}{T}\right)^{2}
$$

and the resistivity $\rho(T)=1 / \sigma(T)$.

The second transport integral is obtained from Eq. (B6) which gives

$$
\begin{aligned}
L_{12} & =\frac{\pi^{2} k_{B}^{2} T^{2}}{3} \Lambda(0, T) \\
& \times\left[\frac{\mathcal{N}_{c}^{\prime}(0)}{\mathcal{N}_{c}(0)}+\frac{\tau^{\prime}(0, T)}{\tau(0, T)}\right] \\
& \simeq-2 L_{11} \frac{\pi^{2} k_{B}^{2} T^{2}}{\tilde{\omega}_{f}} \simeq \mp 4 L_{11} \frac{\pi^{2} k_{B} T^{2}}{n_{f} T_{0}} .
\end{aligned}
$$

In the second equation we used $\tilde{\omega}_{f} \simeq \pm n_{f} k_{B} T_{0} / 2$, which holds for heavy fermions with $n_{f} \simeq 1$ and large $N$ only.

The thermal conductivity is defined by the expression,

$$
\kappa(T)=N \frac{L_{11}}{T}\left[\frac{L_{22}}{L_{11}}-\left(\frac{L_{12}}{L_{11}}\right)^{2}\right]
$$

and follows from the previous results which give $L_{11}=2 \Lambda(0, T) / 3, L_{12} / L_{11} \simeq 2 \pi^{2} k_{B}^{2} T^{2} / \tilde{\omega}_{f}, L_{22}=$ $\pi^{2} k_{B}^{2} T^{2} \Lambda(0, T) / 3$, and $L_{22} / L_{11}=\pi^{2} k_{B}^{2} T^{2} / 2$. In the heavy fermion limit this yields the expression in Eq. (53) used in the main text.
${ }^{1}$ K. Behnia, D. Jaccard, and J. Flouquet, J. Phys.: Condens. Matter 16, 5187 (2004).

2 J. Sakurai and Y. Isikawa, J. Phys. Soc. Japan 74, 1926 (2005).

3 Z. Hossain, C. Geibel, N. Senthilkumaran, M. Deppe, M. Baenitz, F. Schiller, and S. L. Molodtsov, Phys. Rev. B 69, 014422 (2004).

4 J. Sakurai, A. Iwasaki, Q. Lu, D. Ho, Y. Isikawa, J. R. Fernández, and C. G. Sal, J. Phys. Soc. Japan 71, 2829 (2002).

5 M. Očko, D. Drobac, J. L. Sarrao, and Z. Fisk, Phys. Rev. 64, 085103 (2001).

6 M. Očko, J. L. Sarrao, and ` Z. Šimek, J. Mag. Mag. Mater. 43 - 46, 284 (2004).

7 U. Kohler, N. Oeschler, F. Steglich, S. Maquilon, and Z. Fisk, Physical Review B 77, 104412 (2008).

${ }^{8}$ K. Kadowaki and S. B. Woods, Solid State Commun. 71, 1149 (1987).

${ }^{9}$ H. Kontani, J. Phys. Soc. Japan 73, 515 (2004).

10 N. Tsujii, H. Kontani, and K. Yoshimura, Phys. Rev. Lett. 94, 057201 (2005).

11 N. E. Hussey, J. Phys. Soc. Japan 74, 1107 (2005).

12 K. Yamada and K. Yosida, Prog. Theor. Phys. 76, 681 (1986).
13 K. Yamada, Electron Correlation in Metals (Cambridge University Press, Cambridge, 2004).

14 K. Miyake and H. Kohno, J. Phys. Soc. Japan 74, 254 (2005).

15 N. F. Mott and H. Jones, The Theory of the Properites of Metals and Alloys (Dover Publications, London, 1958).

16 C. Grenzebach, F. B. Anders, G. Czycholl, and T. Pruschke, Phys. Rev. B 74, 195119 (2006).

17 A. Georges, G. Kotliar, W. Krauth, and M. J. Rozenberg, Rev. Mod. Phys. 68, 13 (1996).

18 V. Zlatić, R. Monnier, J. Freericks, and K. W. Becker, Phys. Rev. B 76, 085122 (2007).

19 A. Hewson, The Kondo Problem to Heavy Fermions (Cambridge University Press, Cambridge, 1993).

${ }^{20}$ H. Ehrenreich and F. Spaepen, Good Thermoelectrics (Academic Press, San Diego, 1997), vol. 51 of Solid State Physics, chap. G. D. Mahan, p. 81.

21 D. Jaccard, E. Vargoz, K. Alami-Yadri, and H. Wilhelm, Rev. High Pressure Sci. Technol. 7, 412 (1998).

22 A. T. Holems, D. Jaccard, and K. Miyake, Phys. Rev. B 69, 024508 (2004).

23 H. Wilhelm and D. Jaccard, Phys. Rev. 66, 064428 (2002).

${ }^{24}$ H. Wilhelm, D.Jaccard, V. Zlatic, R. Monnier, B. Delley, and B. Coqblin, J. Phys: Cond. Matter 17, S830 (2005). 
${ }^{25}$ G. D. Mahan, Many-Particle Physics (Plenum, New York, 1981).

${ }^{26}$ N. Vidhyadhiraja and D. Logan, European Phys. Jour. B 39, 313 (2004).

27 D. E. Logan and N. S. Vidhyadhiraja, J. Phys: Cond. Matter 17, 2935 (2005).

28 N. Kernavanois, S. Raymond, E. Ressouche, B. Grenier, J. Flouquet, and P. Lejay, Physical Review B 71, 064404 (2005).

${ }^{29}$ K. Miyake and H. Maebashi, J. Phys. Soc. Japan 71, 1007 (2002).
30 V. Zlatić and R. Monnier, Phys. Rev. B 71, 165109 (2005).

31 K. Alami-Yadri, D. Jaccard, and D. Andreica, J. Low. Temp.Phys 114, 135 (1999).

32 S. Burdin and V. Zlatić, unpublished (2008).

33 A. Khurana, Phys. Rev. Lett. 64, 1990 (1990).

${ }^{34}$ V. Zlatić and B. Horvatić, Solid State Commun. 75, 263 (1990).

35 J. K. Freericks and V. Zlatić, Phys. Rev. B 64, 245118 (2001).

36 B. Horvatić and V. Zlatić, Phys. Rev. B 30, 6717 (1984). 